\title{
Segmentação Semântica de Medidores de Energia Elétrica e Componentes de Identificação
}

\author{
Arthur Costa Serra* João Vitor F. França* \\ Jefferson Alves de Sousa ${ }^{*}$ Robherson W. S. Costa* \\ Italo Francyles S. da Silva* Simara Vieira da Rocha* \\ Aristófanes Correa Silva* Anselmo Cardoso de Paiva* \\ Eliana Márcia G. Monteiro ** Italo Fernandes S. da Silva ** \\ Márcia Izabel A. da Silva ${ }^{* *}$ Jose Messias dos Santos ${ }^{* *}$ \\ * Núcleo de Computação Aplicada \\ Universidade Federal do Maranhão (UFMA) - São Luís, MA, Brasil \\ (e-mail: $\{$ arthursrr, jvitorfranca, jefferson.alves, robherson, \\ francyles, simara.rocha, ari, paiva\}@nca.ufma.br) \\ ** Equatorial Energia - São Luís, MA, Brasil \\ (e-mail: \{eliana.monteiro, italo.silva, \\ marcia.silva,jose.messias\}@cemar-ma.com.br)
}

\begin{abstract}
Brazilian Electricity Regulatory Agency (ANEEL) classifies energy consumption measurement and bill processing errors as non-technical losses. When those irregularities are identified, meter imaging and geographic location are requested to a reader. Those images are sent to audit sector. This receives a great volume of images, and its whole analysis is too slow. As an alternative, there is the self-reading, which is the reading done by the customer through digital platforms. To ensure safety in self-reading process, a validation stage (self-audit) is necessary. This work proposes a method based on deep learning for semantic segmentation of energy meters and identification components, aiming to contribute with more efficiency in reading validation. This method obtains $73.10 \%$ mean average precision (mAP) for intersection over union (IoU) $\geq 0.50 ; 42.17 \% \mathrm{mAP}$ for $\mathrm{Iou} \geq 0.75$ and $41.28 \% \mathrm{mAP}$ for $\mathrm{IoU} \geq 0.99$.

Resumo: A Agência Nacional de Energia Elétrica (ANEEL) classifica erros de medição de consumo e processamento de fatura como perdas não-técnicas. Quando essas irregularidades são identificadas, é solicitada a aquisição da imagem do medidor e a captura da localização geográfica do leiturista para registrar a sua presença no local. Essas imagens são enviadas para o setor de auditoria. Este recebe um grande volume de imagens, cuja análise completa é muito lenta. Como alternativa, tem-se a autoleitura, que é a leitura feita pelo próprio cliente através de plataformas digitais. E para garantir a segurança no processo de autoleitura, é necessária uma etapa automática de validação (autoauditoria). Este trabalho propõe um método baseado em aprendizado profundo para a segmentação semântica de medidores de energia e componentes de identificação, almejando contribuir com mais eficiência ao processo de validação de leitura. O método apresenta mean average precision (mAP) de $73,10 \%$ para os casos em que intersection over union $(\mathrm{IoU}) \geq$ a 0,$50 ; 42,17 \%$ para $\mathrm{IoU} \geq 0,75$ e $41,28 \%$ quando $\mathrm{IoU} \geq 0,99$.
\end{abstract}

Keywords: Self-reading, Self-audit, Image Processing, Semantic Segmentation, Deep Learning Palavras-chaves: Autoleitura, Autoauditoria, Processamento de imagens, Segmentação Semântica, Aprendizado Profundo

\section{INTRODUÇÃO}

Medidores de energia elétrica são equipamentos capazes de mensurar o consumo de energia elétrica de uma unidade consumidora. Para efetuar a leitura do consumo de um consumidor, a Companhia Energética do Maranhão (CEMAR) e as Centrais Elétricas do Pará S.A (CELPA)

^ Este trabalho contou com o apoio do projeto Autoleitura, financiado pelo grupo Equatorial Energia, no âmbito do Programa de P \& D da Agência Nacional de Energia Elétrica (ANEEL) No PD-00037$0032 / 2018$. utilizam um dispositivo móvel e uma impressora portátil. Nesse processo, as informações de consumo são coletadas e processadas, gerando, por fim, a fatura impressa dos consumidores. O leiturista precisa informar manualmente o consumo tal qual é mostrado no medidor de energia, o que torna esse procedimento mais lento e passível de erros, os quais acarretam, portanto, em inconsistências no faturamento mensal.

De acordo com a Agência Nacional de Energia Elétrica (ANEEL), erros de medição de consumo e processamento 
de fatura, sejam por falha humana, ou impedimento de acesso à unidade consumidora, são considerados perdas não-técnicas (ANEEL, 2019). Esse tipo de perda é descoberto durante os processos de leitura e fiscalização do consumo de energia. Nos casos em que o consumo aferido para um consumidor está fora da sua média de consumo, ou o leiturista não consegue realizar a leitura, é solicitada a aquisição da imagem do medidor e a captura da localização geográfica do leiturista para registrar a sua presença no local.

Essas informações são enviadas para o setor de crítica das concessionárias, onde se efetuam auditorias para avaliar esses casos. Porém, em decorrência da grande quantidade de imagens recebidas, a auditoria ocorre por amostragem, visto que a avaliação de todos os casos demandaria um grande número de pessoal e de tempo. Em geral, analisa-se nas imagens questões como ausência de medidor, identificação incorreta do medidor, impossibilidade de leitura e consumo anômalo.

Uma alternativa para auxiliar neste processo, seria a leitura realizada pelo próprio consumidor, denominada autoleitura. Esta engloba o uso de plataformas digitais, como sites ou aplicativo desenvolvido para dispositivo móvel, por onde o consumidor registraria e enviaria as informações de consumo. Este processo traz vantagens ao consumidor, pois lhe garante um controle maior sobre o seu consumo de energia; e para as concessionárias, porque proporciona a diminuição de custos e a redução dos erros de leituras.

Nesse contexto, métodos computacionais capazes de realizar a leitura automática do consumo de energia através da análise de imagem podem facilitar e agilizar a mensuração do consumo, tornando-a menos suscetível a erros. E para garantir a segurança no processo de autoleitura, esse método precisa contar com uma etapa automática de auditoria, denominada de autoauditoria, a ser executada periodicamente ou quando o consumo aferido apresentar valores fora da faixa mensal esperada para um consumidor.

Inicialmente, a imagem é adquirida pelo consumidor. Em seguida é enviada para o servidor da concessionária onde será feito todo o tratamento da imagem e identificação dos componentes do medidor. A primeira etapa do processo é identificar se existe ou não medidor na imagem. Caso exista, este é extraído da imagem. O próximo passo é a segmentação dos seguintes componentes: o display, onde estão os dígitos do consumo e o código de identificação do medidor $(t a g)$.

Embasando-se nessa proposta, este trabalho apresenta um método para a segmentação semântica de medidores de energia elétrica e componentes de identificação, aplicado em imagens dos medidores utilizados pelas concessionárias CEMAR e CELPA, objetivando contribuir com mais eficiência ao processo de validação de leitura e também com a diminuição de perdas relacionadas ao faturamento, o que poderá garantir segurança para consumidores e para as concessionárias.

Na literatura, são encontrados alguns trabalhos com propostas voltadas para a leitura de consumo automática baseada em imagens. De maneira geral, esses trabalhos utilizam câmeras fixadas em frente ao medidor para adqui- rir a imagem da região do display e, em seguida, utilizar processamento de imagem e aprendizado de máquina para realizar a leitura através do reconhecimento de dígitos (Parthiban and Palanisamy, 2013; Zhang et al., 2016).

Ainda no contexto de leitura automática, Reis et al. (2017) propuseram um método para segmentar automaticamente a região do medidor de energia elétrica em imagens adquiridas por leituristas de distribuidoras de energia, de forma a facilitar o processo de auditoria com a validação da existência de medidor. O método utiliza binarização e estatística para delimitar a região do medidor na imagem, obtendo acurácia de $96,41 \%, 89,81 \%$ e 78,85\%, respectivamente para as imagens categorizadas como boas, regulares e ruins.

Diferente desses trabalhos relacionados, nossa proposta é baseada em técnicas aprendizado profundo aplicadas à tarefa de segmentação semântica, com o intuito de detectar, nas imagens, o medidor e os componentes de identificação. Esse método é parte integrante de uma aplicação, em desenvolvimento, para a implantação dos processos de autoleitura e autoauditoria.

Este trabalho está estruturado da seguinte forma: a Seção 2 apresenta detalhes sobre o conjunto de imagens utilizado e explica o método proposto; a Seção 3 discute os resultados obtidos por este estudo; e, a Seção 4 apresenta as conclusões.

\section{MATERIAIS E MÉTODOS}

Este seção descreve os procedimentos realizados para a segmentação semântica de medidores de energia e componentes de identificação. Também são apresentados detalhes sobre o conjunto de imagens utilizado para validar o método proposto.

\subsection{Base de imagens}

Os medidores de energia utilizados pelas companhias CEMAR e CELPA são de dois tipos: digitais e analógicos. No geral, os medidores são protegidos por uma caixa com uma parte transparente para que o leiturista possa conferir o código de identificação (tag) e os dígitos no display. A Figura 1 mostra exemplos de imagens que compõem a base.

A base tem, ao todo, 4325 imagens. Essas foram capturadas sob diferentes condições, simulando o processo de auditoria no qual, em alguns casos, quem captura a imagem pode não ter familiaridade com a tecnologia utilizada, ou a iluminação do ambiente não está favorável; ou quando a caixa protetora apresenta condições precárias devido itempéries. Sendo assim, a base é também composta por imagens borradas, com oclusões parciais e diferentes orientações. Há também imagens sem a presença do medidor.

Na Figura 2, os medidores estão destacados pelo retângulo número 1; os retângulos 2 e 3 delimitam, respectivamente, as regiões da tag e do display. Cada imagem está associada a um arquivo com anotações manuais dos pontos $\mathrm{x}, \mathrm{y}$, $\mathrm{w}, \mathrm{h}$ que determinam os limites das bounding boxes que localizam o medidor, a tag e o display, objetos de interesse do método de segmentação. 

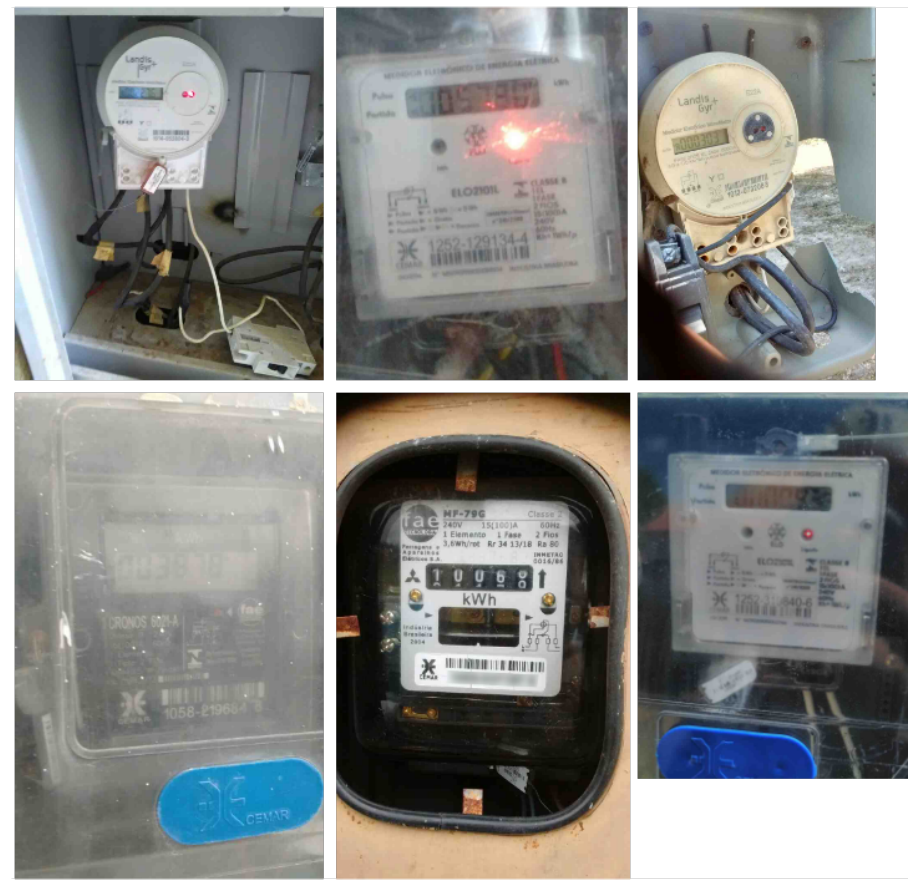

Figura 1. Exemplos de imagens da base.

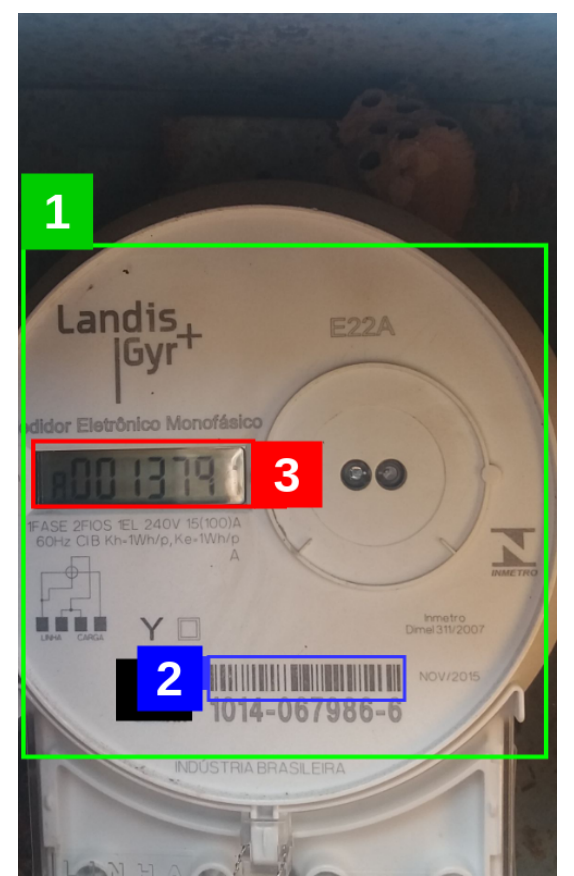

Figura 2. Regiões do medidor (1), tag (2) e display (3).

2.2 Segmentação Semântica utilizando a Rede Single Shot Multibox Detector

Este trabalho tem como objetivo a segmentação semântica de medidores e componentes de identificação. Esta etapa surge como o primeiro passo para a auditoria por imagem, pois, para esse processo, a identificação e segmentação dessas regiões é imprescindível.

Para esta tarefa, é proposto o uso de uma arquitetura de rede neural chamada Single Shot Multibox Detector (SSD). Essa rede tem apresentado resultados promissores para a detecção e segmentação de objetos em imagens
(Liu et al., 2015). E também, segundo Huang et al. (2017), é um modelo capaz de gerar bons resultados balanceando velocidade, acurácia e uso de memória. A Figura 3 apresenta o fluxo de execução do método.

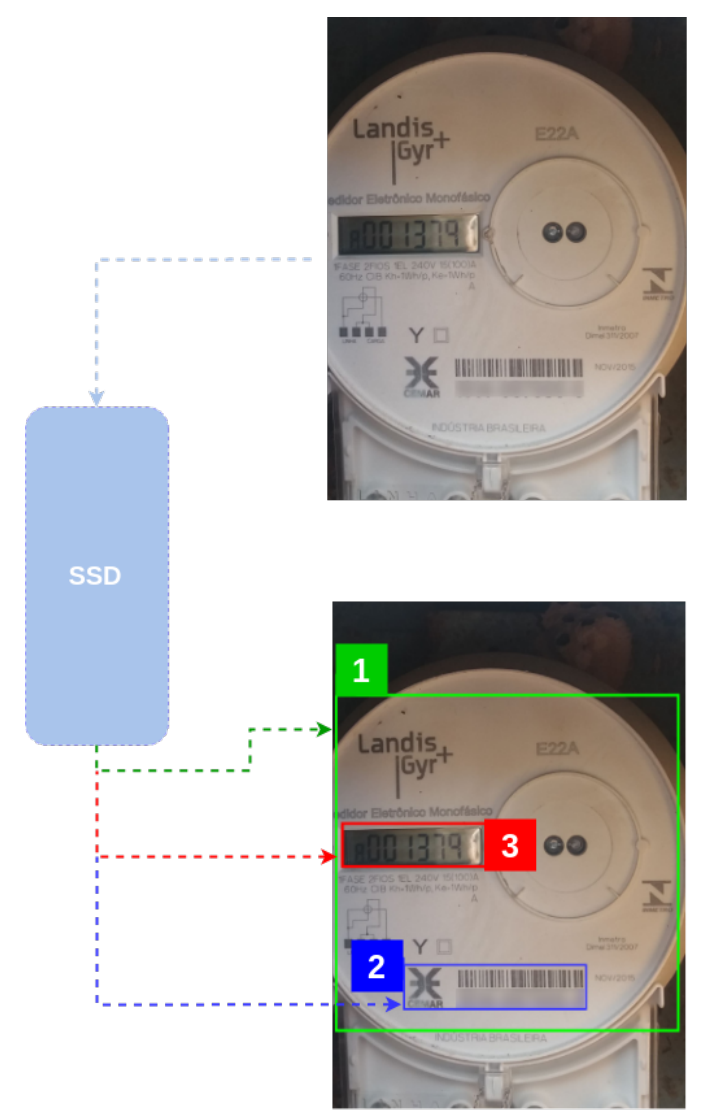

Figura 3. Etapas do Método Proposto

A entrada para a rede SSD é a imagem capturada. Essa rede, então, procura na imagem a localização do medidor (1), tag (2) e display (3) e, por fim, delimita as regiões à medida que identifica a presença desses objetos de interesse na imagem.

Desenvolvida por Liu et al. (2015), a Single Shot Multibox Detector (SSD) é uma rede para detecção de objetos em imagens em uma única etapa. Ela trabalha sobre amostras densas de pixels que são possíveis regiões. Assim, a SSD consegue ser mais rápida se comparada a outras redes que utilizam a abordagem de detecção em duas etapas, como a Faster R-CNN (Ren et al., 2015).

A SSD é uma rede convolucional feed-forward que gera um conjunto fixo de bounding boxes para cada classe de objeto a ser detectado. Ela pode ser dividida em duas fases: de extração de características e de detecção. $\mathrm{Na}$ primeira fase, as camadas são baseadas em alguma arquitetura de rede, como, por exemplo, a VGG (Simonyan and Zisserman, 2014) ou YOLO (Redmon et al., 2015), da qual se elimina a camada responsável pela classificação. Essa rede escolhida é chamada de rede base. A segunda fase é caracterizada pela adição à rede base de várias camadas de convolução, que aplicam filtros de dimensões específicas e geram um número $k$ de bounding boxes, definindo, portanto, a arquitetura final SSD, como ilustrado pela Figura 4. 


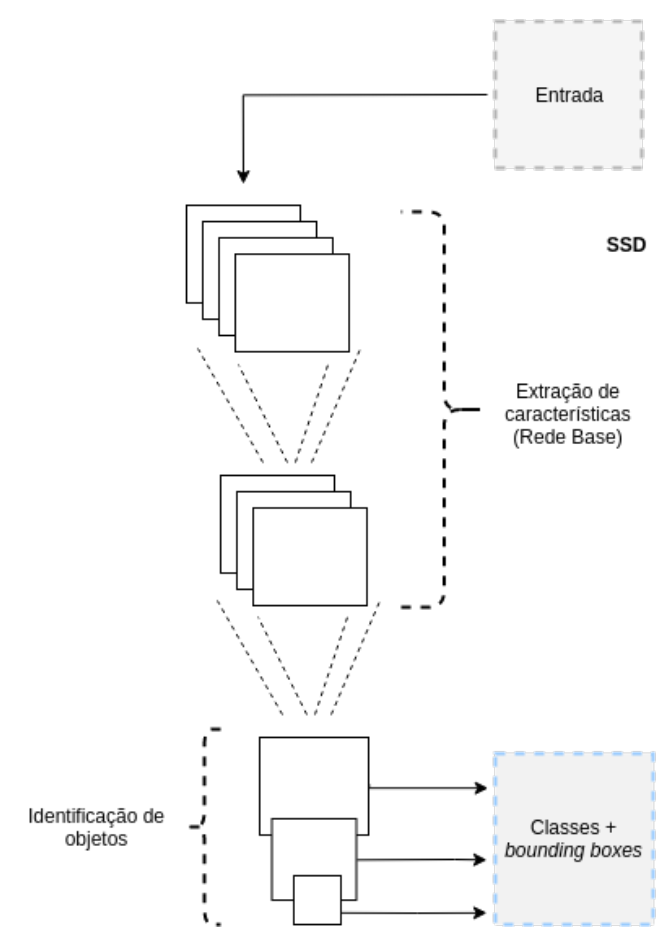

Figura 4. Arquitetura da rede SSD.

No treinamento, a SSD recebe uma imagem de entrada e as bounding boxes de cada objeto. No estágio convolucional, são avaliados um conjunto pequeno de bounding boxes de diferentes aspect ratios e escalas nos vários mapas de características gerados. Então, para cada objeto, são preditas as coordenadas de sua bounding box e também um valor de confiança. A bounding box que delimita corretamente o objeto de interesse é tratada como positiva e as demais, que não o delimitam, como negativas.

No presente trabalho, são adicionadas 33 camadas convolucionais no final da rede base para a fase de predição de bounding boxes para a identificação de objetos. O referido número de camadas foi definido empiricamente. A Tabela 1 mostra mais detalhes sobre essas camadas, na ordem que foram adicionadas.

Tabela 1. Quantidade de camadas convolucionais adicionadas e suas respectivas dimensões.

\begin{tabular}{ccc}
\hline Ordem & Num. camadas & Dimensões \\
\hline 1 & 4 & $19 \times 19$ \\
2 & 15 & $10 \times 10$ \\
3 & 4 & $5 \times 5$ \\
4 & 4 & $3 \times 3$ \\
5 & 4 & $2 \times 2$ \\
6 & 2 & $1 \times 1$ \\
\hline
\end{tabular}

Uma vez que a rede encontra, na imagem, a localização do medidor, da $t a g$ e do display, basta fazer o recorte desses componentes utilizando as bounding boxes geradas pela SSD.

\subsection{Métricas de Avaliação}

São utilizadas como métricas de avaliação dos resultados produzidos pelo método a intersection over union (IoU) e Mean Average Precision (mAP).
O IoU mede a sobreposição entre duas regiões definidas pelas bounding boxes, a gerada pelo método e a anotada manualmente (ground-truth)). Calcula-se pela razão entre a interseção dos conjuntos X e Y e a união desses (Equação 1). Neste caso, X e Y representam as bounding boxes.

$$
I o U=\frac{|X \cap Y|}{|X \cup Y|}
$$

A Average Precision (AP) é estabelecida como a área sob a curva definida pela relação entre Precision (P) e Recall (R), calculados como mostram as Equações 2 e 3

$$
\begin{aligned}
R & =\frac{V P}{V P+F N} \\
P & =\frac{V P}{V P+F P}
\end{aligned}
$$

onde VP e FP são os verdadeiros e falsos positivos, respectivamente; e FN os falsos negativos.

No caso da AP, é preciso estabelecer uma condição para que a Precision seja considerada correta. Essa condição, neste caso, é se a IoU obtida é igual ou superior a algum valor definido.

Logo, mAP é definida pela média da Average Precision calculada para cada classe de objeto. Essa métrica apresenta valores entre 0 e 1 (100\%). Quanto mais próximo de 1 , melhor é o resultado.

\section{RESULTADOS E DISCUSSÃO}

A rede SSD foi implementada em linguagem Python $3.7^{1}$ com o auxílio da biblioteca TensorFlow ${ }^{2}$, que é voltada para aplicações de aprendizado de máquina. A SSD foi treinada por 50 épocas em uma GPU NVIDIA Tesla K80 utilizando o otimizador rmsprop (Tieleman and Hinton, 2012). Cada época contém $200 \mathrm{mil}$ passos. A taxa de aprendizado utilizada foi de $4 \times 10^{-3}$. Demais parâmetros de configuração seguem o padrão estabelecido pelo TensorFlow.

A base de imagens foi dividida em $70 \%$ para treino e $30 \%$ para teste. Os resultados produzidos pelo método proposto podem ser visualizados pela Tabela 2.

Tabela 2. Resultados obtidos pelo método proposto.

\begin{tabular}{cc}
\hline Critério & mAP \\
\hline IoU $\geq 0,50$ & $73,10 \%$ \\
IoU $\geq 0,75$ & $42,17 \%$ \\
IoU $\geq 0,99$ & $41,28 \%$ \\
\hline
\end{tabular}

Para avaliar a mAP obtida pelo método, é preciso também analisar o critério baseado em IoU para a consideração de predições corretas. No caso em que IoU $\geq 0,50$, o método alcança $73,10 \%$ de mAP, portanto, pode-se verificar que os resultados de AP para as classes medidor, tag e display estão razoavelmente equilibrados. Ou seja, os objetos de interesse são identificados corretamente em grande parte das imagens com IoU igual ou superior a 0,50.

\footnotetext{
1 https://www.python.org/

2 https://www.tensorflow.org/
} 
Para o limiar de $\mathrm{IoU} \geq 0,75$, o valor de $\mathrm{mAP}$ diminui para $42,17 \%$. E quando se define $\mathrm{IoU} \geq 0,99$, o resultado de $\mathrm{mAP}$ alcança $41,28 \%$. Observa-se que, conforme IoU se eleva, o valor de mAP diminui. Isso se dá porque a avaliação das predições torna-se mais criteriosa, logo, as segmentações serão descartadas caso o seu valor IoU esteja fora da faixa de valores desejada.

No entanto, o fato de as bounding boxes geradas pela rede não alcançarem, em grande totalidade, $100 \%$ de IoU não significa que os objetos não tenham sido identificados na imagem. Esse resultado é bastante sensível às anotações manuais, visto que elas são utilizadas para o cálculo das métricas de avaliação.

A Figura 5 mostra exemplos de imagens submetidas ao método, nas quais as regiões de interesse foram delimitadas corretamente.

As Figuras 5(A), 5(B) foram capturadas próximas ao medidor. Na primeira, o método obteve uma taxa de confiança mais baixa para o medidor em comparação aos componentes, devido a proximidade da câmera, o que fez a rede perder informações de contexto. No caso da 5(B), mesmo apresentando inclinação, as regiões são delimitadas. Outra observação é que devido as diferenças entre os modelos de medidor de energia, a localização do código identificador também varia. Não obstante, o método consegue localizar os objetos de interesse e definir para eles uma bounding box respectiva.

O caso apresentado pela Figura $5(\mathrm{C})$ retrata a aplicação do método em um cenário menos favorável para acertos. Pois, a caixa protetora apresenta condições precárias e isso é um complicador para a visualização do medidor e de seus componentes. No entanto, houve a delimitação correta das regiões de interesse, com destaque para a identificação do display, visto que esse se encontra numa região de difícil compreensão na imagem.

Por fim, a Figura 5(D) apresenta um caso capturado com certa distância, o que oferece mais informação de contexto à rede, resultando em uma alta taxa de confiança para a delimitação do medidor, display e tag.

Como observado, os resultados obtidos mostram-se satisfatórios, pois, mesmo em condições adversas, o método mostrou-se capaz de delimitar as regiões dos medidores e componentes.

\section{CONCLUSÃO E TRABALHOS FUTUROS}

Este trabalho apresentou um método para a segmentação semântica de medidores de energia elétrica e componentes de identificação. O trabalho propôs a utilização da rede convolucional SSD aplicada à delimitação das regiões do medidor, do display e do código identificador (tag).

O método apresenta mAP de $73,10 \%$ para os casos em que a métrica IoU é igual ou superior a 0,50; 42,17\% para Iou $\geq 0,75$ e $41,28 \%$ quando IoU $\geq 0,99$. Esses resultados considerados são vistos como promissores para a aplicação ao qual este trabalho será integrado. Entretanto, entendese que podem ser melhorados com o desenvolvimento de mais testes.

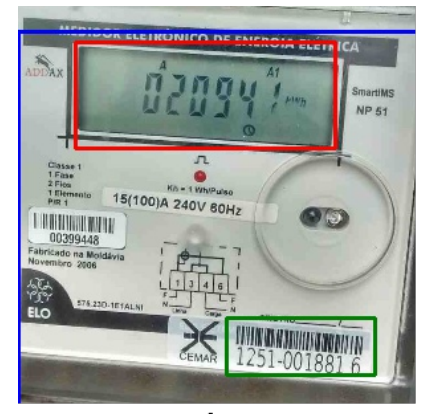

A
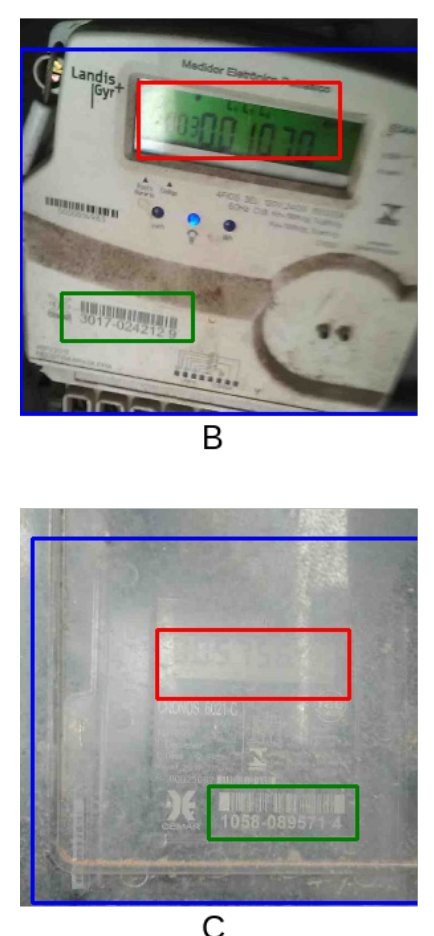

C

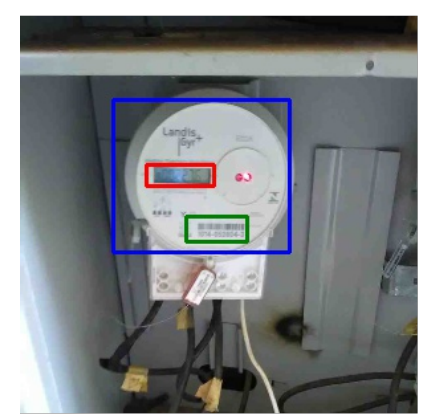

D
Medidor: 56,50\% Display: 97,60\% Tag: 98,10\%

Medidor: $59,50 \%$

Display: $59,20 \%$

Tag: 57,30\%

Medidor: $72,00 \%$

Display: 97,20\%

Tag: 57,40\%

Medidor: 99,50\%

Display: $77,70 \%$

Tag: 83,40\%
Figura 5. Segmentação semântica do medidor e componentes em imagens utilizando o método proposto.

Como trabalho futuro, propõe-se desenvolver o reconhecimento dos dígitos mostrados pelo display e do código de identificação do medidor.

\section{REFERENCIAS}

ANEEL (2019). Energia no brasil e no mundo. URL http: //www2. aneel.gov.br/arquivos/pdf/atlas_ par1_cap2.pdf. 
Huang, J., Rathod, V., Sun, C., Zhu, M., Korattikara, A., Fathi, A., Fischer, I., Wojna, Z., Song, Y., Guadarrama, S., and Murphy, K. (2017). Speed/accuracy tradeoffs for modern convolutional object detectors. In The IEEE Conference on Computer Vision and Pattern Recognition (CVPR).

Liu, W., Anguelov, D., Erhan, D., Szegedy, C., Reed, S.E., Fu, C., and Berg, A.C. (2015). SSD: single shot multibox detector. CoRR, abs/1512.02325. URL http://arxiv . org/abs/1512.02325.

Parthiban, K. and Palanisamy, A. (2013). Reading values in electrical meter using image processing techniques. In Intelligent Interactive Systems and Assistive Technologies (IISAT), 2013 International Conference on, 1-7. IEEE.

Redmon, J., Divvala, S.K., Girshick, R.B., and Farhadi, A. (2015). You only look once: Unified, real-time object detection. CoRR, abs/1506.02640. URL http: //arxiv . org/abs/1506.02640

Reis, A.B.S., de Sousa, J.A., da Silva Neto, O.P., Silva, A.C., de Paiva, A.C., de Almeida, J.D.S., Junior, G.B., Azevedo, L.M., and Monteiro, E.M. (2017). Segmentação automática de medidores eletromecânico e eletrônico baseado em imagens. In Simpósio Brasileiro de Automação Inteligente (SBAI).

Ren, S., He, K., Girshick, R.B., and Sun, J. (2015). Faster R-CNN: towards real-time object detection with region proposal networks. CoRR, abs/1506.01497. URL http: //arxiv.org/abs/1506.01497.

Simonyan, K. and Zisserman, A. (2014). Very deep convolutional networks for large-scale image recognition. arXiv 1409.1556 .

Tieleman, T. and Hinton, G. (2012). Lecture 6.5RmsProp: Divide the gradient by a running average of its recent magnitude. COURSERA: Neural Networks for Machine Learning.

Zhang, Y., Yang, S., Su, X., Shi, E., and Zhang, H. (2016). Automatic reading of domestic electric meter: an intelligent device based on image processing and zigbee/ethernet communication. Journal of Real-Time Image Processing, 12(1), 133-143. 